\title{
MEDICO LEGAL KNOWLEDGE AND ATTITUDE AMONG MEDICAL STUDENTS REGARDING EYE DONATION.
}

VinayKumar. M. S.

1. Assistant Professor, Department of Forensic Medicine, Prathima Institute of Medical Sciences.

\section{CORRESPONDING AUTHOR}

Dr. Vinay Kumar M. S.

H no 302, C block, Pims Campus ,

Karimnagar, Andhra Pradesh,

E-mail: drvinaykumarms2006@gmail.com

Ph: 00919985633244

ABSTRACT:In developing countries like India, shortage of organ donors is a fundamental public health problem with medical and legal implications. Improving the awareness, medico-legal knowledge and attitude among medical students who are considered as the future health care providers of the community could help to promote organ donation. The objective of the present study was to assess the level of medico-legal knowledge and attitude of second year medical students on eye donation. One hundred and twenty two medical students participated in the study. The awareness and medico-legal knowledge which included procedure on eye donation, time factor, valid consent, place of contact and the reasons for willingness and unwillingness to donate eyes were assessed through pretested structured questionnaire. 99\% of the medical students were aware of eye donation.59\% of the participants responded that the eyes should be removed within 6 hours of death and $81.9 \%$ responded that the donor should be consented. However, only $18.03 \%$ responded that a valid consent should be obtained from the relatives or legal heirs after the death of the donor. $89.3 \%$ of the students were willing to donate the eyes and only $10 \%$ were unwilling. Nobility (64\%) was the main motivational factor for eye donation and those who were unwilling attributed it to religious (84.6\%) and cosmetic (15.4\%) factors. Second-year medical students have a good knowledge regarding eye donation but their knowledge on valid consent of legal heir and relatives and the knowledge on newer legislation like presumed consent and required request needs to be improved which can play a significant role in eye donation movement in the country.

KEYWORDS: Eye donation, Medical students, Medico-legal knowledge.

INTRODUCTION: Corneal diseases are a significant cause of visual impairment and blindness in the developing world. Approximately 18.7 million people are blind in India and 190,000 are blind from bilateral corneal disease. For such people, corneal transplantation helps in restoring their sight. According to the Eye Bank Association of India, the current cornea procurement rate in India is22,000 per year. [1-3] However, the current corneal procurement rates are inadequate to meet the transplantation need in India. Despite the reasonable awareness campaigns, the knowledge on eye donation is very poor. The medical students are the future health care providers for the community, having strong scientific base and an inherent inclination to serve the mankind. Medical professionals can enhance eye donation rates by educating and motivating the relatives in case of patient's death. .. Materials and Methods A cross-sectional study was carried out in the Department of Forensic Medicine, Prathima 
Institute of Medical Sciences, Karimnagar.The study sample included 122 second year medical students. They were assessed for their awareness, medico legal knowledge and attitude regarding eye donation with the help of a scientifically designed pre-tested, structured questionnaire. Analysis were made and conclusions were drawn regarding the awareness, knowledge on procedure, time of donation, place of contact, valid consent of donor and legal heir (relatives) and their willingness to donate the eyes.

RESULTS: One hundred and twenty two second year medical students participated in the present study. Among them 43 (35.25\%) were males and 79 (64.75\%) were females.99.1\% of the medical students were aware of eye donation and $81.9 \%$ were aware of corneal transplantation (table 1).59\% of the participants responded that the eyes should be removed within 6 hours of death (table 2), 81.9\% responded that the donor should be consented for eye donation (table 3). However, only $18.03 \%$ responded that a valid consent should be obtained from the relatives or legal heirs after the death of the donor (table 3). 89.3\% of the students were willing to donate the eyes and only $10 \%$ were unwilling (table 4). Nobility (64\%) was the main motivational factor for eye donation (table 5) and those who were unwilling attributed it to religious (84.6\%) and cosmetic (15.4\%) factors (table 6).

DISCUSSION: In the present study, $99.1 \%$ of the medical students were aware about eye donation. This is consistent with other studies, where $99.4 \%$ and $98.4 \%$ of first-year medical students in Delhi and Bangalore and $96.8 \%$ of first and second year nursing students in Bangalore were aware of eye donation. [2,3] The awareness on eye donation is much lower in the South Indian population (50.7\%), ${ }^{[4]}$ as compared to urban population of Hyderabad (73.8\%) ${ }^{[5]}$ and rural population of Andhra Pradesh (30.7\%). [6] During eye donation, the whole eye or the cornea issurgically removed from a deceased person, and then the cornea is processed and grafted to a needy person.[7] In the present study, $81.9 \%$ of the participants were aware about corneal transplantation. This is consistent with the study on medical students in Delhi, where the awareness was $86.1 \%{ }_{.[2]}$ However, it is poor in the general population $(20.5 \%) .{ }^{4}$ From the above observations, it is evident that the awareness is less in the general population when compared to medical students.

The success of eye donation lies in removing and storing the eyes at the right time. Corneas can be removed up to 12 hours after death, but ideally within 6 hours. ${ }^{[7]}$ In the present study, 59\% of the participants responded that less than 6 hours was the ideal time for eye collection. This differs from other studies, where $91 \%$ of the medical students in Bengaluru, [12] $41 \%$ of the medical students in Delhi [2] were aware that eye donation had to be done within 6 hours of death. Whole eyes can be stored in a moist chamber at $2-8^{\circ} \mathrm{C}$, which has to be used within 48 hours. Excised corneas can be stored in a preservative media such as McCary Kaufman medium (MK medium) or optisol, both maintained at $4^{\circ} \mathrm{C}$. Corneas can be stored for 96 hours in the MK medium and 10 days in optisol. [7]

For eye donation, one should be aware about contacting the right place with facilities for removal and storage of the eye or the cornea. About $80 \%$ of the present study group knew about the correct place of contact for eye donation such as Medical colleges, eye banks and other health care facilities like Ophthalmic clinics. This differs from other studies where only $27.2 \%$ of the medical students in Delhi and $32.9 \%$ of the nursing students knew about the contact place 
for eye donation. [2, 3] Eye banks are the places where the donor corneas are harvested, processed and then distributed for the purpose of corneal grafting. [8]

On assessing the knowledge on giving consent for eye donation, the present study group mentioned donor as the right person to give consent (81.9\%), and relatives of the donor should give consent after death of the donor (18.03\%). Though the donor has pledged his/her eyes during life time, it is essential to obtain consent from the nearest relatives or the legal heirs after the death of the donor. ${ }^{[9]}$ Only $0.8 \%$ of present study group had an idea about Presumed consent and Required Request. Many countries like Spain have opted for change in legislationand introduced a presumed consent, whereby organs can be used for transplantation after deathunless individuals have objected during their lifetime. The presumed consent law or

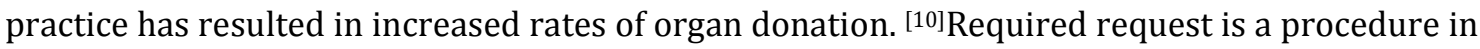
which hospitals are made mandatory to ask for consent for eye donation to the legal heirs or relatives of the deceased person.

About $89 \%$ of the present study groups were willing to donate their eyes whereas only32.9\% of rural population of Andhra Pradesh [6] was willing to donate. The main motive for eye donation among the medical students was nobility (64.2\%), followed by helping the blind (25.6\%), setting an example (6.4\%) and popularity (3.6\%). This is consistent with other studies, where nobility was the major motivational force for eye donation among medical students in Delhi and the nursing students of Bangalore. ${ }^{[2,3]}$ Only $11 \%$ of medical students were not willing for eye donation. The reasons for not pledging eyes were unacceptable idea (Religious) of separating the eyes from the body (84\%) and disfigurement (cosmetic) of face (16\%). It is important to mention that neither enucleation nor corneal excision causes disfigurement of the face or any delay in funeral arrangements. Moreover, the socioeconomic factors such as education, marital status, residential area and situational factors such as the cause of death also play an important role on the willingness to consent for donation. [11]

CONCLUSION: Medical students of second-year MBBS were assessed for their awareness, medico-legal knowledge and attitude on eye donation.

1. The awareness of eye donation among these medical students is higher compared to general population.

2 Majority of these students had correct knowledge about procedure, time factor and place of contact for eye donation.

3 Majority of them had right knowledge about valid consent of the donor, however their knowledge about the consent of legal heirs or the relatives of the deceased after the death of the donor was low.

4 A very few of them had the knowledge about newer legislations of Presumed consent and Required request, although these are still not implemented in India.

5 Majority of the students were willing to donate their eyes, only a small percentage of students who were not willing attributed it to religious and cosmetic factors.

Being the future health care providers, the medical students need to educate and motivate the general population, their friends and relatives about eye donation in order to overcome the backlog of corneal transplantation in this developing nation INDIA. 


\section{REFERENCES:}

1. Whitcher JP, Srinivasan M, Upadhyay MP. Corneal blindness: A global perspective. Bull World Health Organ 2001; 79:214-221.

2. Singh MM, Rahi M, Pagare D, Ingle GK. Medical students' perception on eye donation in Delhi. Indian J Ophthalmol 2007; 55:49-53.

3. Gupta A, Jain S, Jain T, Gupta K. Awareness and perception regarding eye donation in students of a nursing college in Bangalore. Indian J Community Med 2009; 34:122-125.

4. Priyadarshini B, Srinivasan M, Padmavathi A, Selvam S, Saradha R, Nirmalan PK. Awareness of eye donation in an adult population of southern India: a pilot study. Indian J Ophthalmol 2003; 51:101-104.

5. Dandona R, Dandona L, Naduvilath TJ, McCarty CA, Rao GN. Awareness of eye donation in an urban population in India. Aust N Z J Ophthalmol 1999; 27:166-169.

6. Krishnaiah S, Kovai V, Nutheti R, Shamanna BR, Thomas R, Rao GN. Awareness of eye donation in the rural population of India. Indian J Ophthalmol 2004; 52:73-78.

7. Rao GN, Gopinathan U. Eye banking: an introduction. Community Eye Health J 2009; 22:46-47.

8. Rao GN. What is eye banking? Indian J Ophthalmol 1996; 44:1-2.

9. Karthi LP, Agnihotri AK. Corneal transplants - an overview with an emphasis on current scenario in Mauritius. Internet J Med Update 2007; 2:18-21.

10. Rithalia A, McDaid C, Suekarran S, Myers L, Sowden A. Impact of presumed consent for organ donation on donation rates: a systematic review. BMJ 2009; 338:a3162.

11. Krieglstein TR, Neubauer AS, Welge-Lüssen U, Priglinger S, Kampik A, Priemer F. Cornea donation: factors influencing consent. Ophthalmologe 2001; 98:545-549.

\section{OBSERVATIONS:}

\section{Table 1: Awareness on eye donation, corneal transplantation and presumed consent}

\section{Awareness}

Eye donation

Corneal transplant

Presumed consent/ Required request
Frequency

$121(99.1 \%)$

$100(81.9 \%)$

$1(0.8 \%)$

\section{Table 2: Knowledge on time of eye donation}

Time

$<6$ hours after death

$>6$ hours after death
Frequency

72(59.1\%)

$50(40.9 \%)$ 
Table 3: Consent for eye donation.

Consent

Frequency

Only Donor

$100(81.9 \%)$

Both donor and Relative (legal heir)

$22(18.03 \%)$

after death of donor

Table 4: Attitude on eye donation

Attitude

Frequency

Willing

$109(89.3 \%)$

Unwilling

$13(10.7 \%)$

Table 5: Reasons for eye donation.

Reasons

Frequency

Noble work

$70(64.2 \%)$

Helping the blind

$28(25.6 \%)$

Set an example

$7(6.4 \%)$

Popularity

$4(3.6 \%)$

Table 6: Reasons for unwillingness for eye donation

Reasons

Frequency

Religious

$11(84.6 \%)$

Cosmetic

$2(15.4 \%)$ 